\title{
Efecto del tiempo de corte y maduración del ensilaje de botón de oro (Tithonia diversifolia) en la digestibilidad in vivo, utilizado como suplemento en ovinos
}

\section{Effect of time of court and maturing of the silage of Tithonia diversifolia in the in vivo digestibility, used as supplement in sheep}
Gutiérrez Castro Litsy ${ }^{1}$, Güechá Castillo Andrea ${ }^{1}$; Céspedes Sanabria Daniel ${ }^{2}$ y Roa Vega María Ligia ${ }^{3}$
${ }^{1}$ Medico Veterinario Zootecnista Universidad de los Llanos; ${ }^{2} \mathrm{MVZ}$. Esp. (c)MSc. Docente UNILLANOS y ${ }^{3} Z$. MSc Docente UNILLANOS

\author{
litsy.gutierrez@unillanos.edu.co
}

Recibido 15 de Abril 2014, Aceptado 11 de Agosto 2014

\section{RESUMEN}

En la zona tropical de Colombia, la nutrición constituye el factor de mayor influencia en procesos productivos y reproductivos de rumiantes, por lo tanto, el objetivo de este trabajo fue evaluar el efecto de la edad de corte de botón de oro Tithonia diversifolia, (Td) y el grado de maduración de su ensilaje sobre la calidad nutricional y digestibilidad in vivo en ovinos criollos de pelo, como suplemento de una dieta base de king grass (PP) (Pennisetum purpureum). Se realizó una poda de nivelación, luego tres cortes: 45, 60 y 75 días, los ensilajes se mezclaron melaza 25\%: ensilaje de Td (ETd) $75 \%$. Para la digestibilidad se emplearon nueve ovinos con peso promedio $30 \mathrm{~kg} \pm 5 \mathrm{~kg}$, a los que se les suministró PP a voluntad y 400 gramos de materia seca (MS) de ETd. Las dietas y excretas fueron evaluadas en laboratorio de Nutrición. Se usó un diseño completamente al azar, de nueve tratamientos que conciernen a los tres cortes y tres tiempos de maduración del ETd: 30, 45 y 60 días, con tres repeticiones. Cuando fue realizado el trabajo de campo y de laboratorio los valores de la calidad nutricional del ensilaje, fueron en promedio: MS $(21,7 \%)$, proteína (12\%), y contenidos de fibra detergente neutro (FDN) por debajo de $40 \%$. Las digestibilidades aparente de MS (DAMS) fueron superior al $60 \%$ con un promedio $68,5 \% \pm 5,5$. Referente a la digestibilidad de la 
proteína cruda (DPC), esta se ve afectada conforme incrementa el tiempo de corte, pero un mayor tiempo de maduración del ETd mantienen o incrementa su digestibilidad, resultando en promedio: $62,3 \% \pm 9,9$.

Palabras clave: Ovino, digestibilidad in vivo, ensilaje, Tithonia diversifolia.

\begin{abstract}
In the tropical zone of Colombia, nutrition is the most influential factor of productive and reproductive processes of ruminant, therefore, the objective of this work was to evaluate the effect of cut age of Tithonia diversifolia (Td) and the degree of maturity of silage on the nutritional quality and digestibility in vivo in crossbred sheep hair as a supplement to a basal diet of King grass (Pennisetum purpureum) (PP). Leveling pruning was done after three cuts: 45, 60 and 75 days, 25\% silages were mixed: $75 \%$ molasses (TdS). For digestibility nine sheep were used with average weight $30 \mathrm{~kg} \pm 5 \mathrm{~kg}$, were fed adlibitum and PP $400 \mathrm{~g}$ dry matter (DM) of TdS. Diets and excreta were evaluated in laboratory Nutrition. Completely randomized design, nine treatments concerning the three cuts and three maturation TdS was used: 30, 45 and 60 days, with three replications. When conducted fieldwork and laboratory values of the nutritional quality of silage were on average: MS (21.7\%), protein (12\%), and content of neutral detergent fiber (NDF) below $40 \%$. Apparent digestibility of DM (DAMS) was higher than $60 \%$ with an average $68.5 \pm 5.5 \%$. Regarding the digestibility of crude protein (CPD), as this is affected increases cutting time, but a longer maturation TdS maintain or increase its digestibility, resulting in average: $62.3 \pm 9.9 \%$.
\end{abstract}

Keywords: Sheep, in vivo digestibility, silage, Tithonia diversifolia.

\title{
INTRODUCCIÓN
}

Países tropicales como Colombia se caracterizan por marcadas fluctuaciones en la cantidad y calidad de las pasturas, que traen como consecuencia una baja productividad en los animales. Esto hace que sea necesario evaluar forrajes adaptados y de buena calidad que provean mayores aportes nutricionales. Los 
forrajes tropicales se utilizan en mayor o menor proporción como fuente 0 alternativa de alimento para animales, con dependencias agroclimáticas y de oportunidad de uso por parte del productor (Valencia et al., 2010).

Teniendo en cuenta los estudios realizados en botánica, fitoquímica y calidad nutricional de Tithonia diversifolia como una especie promisoria en la alimentación de rumiantes sería conveniente integrarla en sistemas de producción animal, ya que existen evidencias como lo reportado por Wanjau et al., (1998), donde especies de plantas no leguminosas como Tithonia diversifolia acumulan cantidades considerables de nitrógeno en sus hojas como las leguminosas, además tiene altos niveles de fósforo, gran volumen radicular, habilidad especial para recuperar los escasos nutrientes del suelo, amplio rango de adaptación, tolera condiciones de acidez y baja fertilidad en el suelo, es muy agresiva y puede soportar la poda a nivel del suelo y la quema, manteniendo su rápido crecimiento que demanda pocos insumos para su cultivo (Ríos, 1999), las anteriores características ratifican a esta especie como una opción para la alimentación en sistemas de producción animal (Mahecha y Rosales, 2005).

El uso del botón de oro como suplemento en animales es cada vez más generalizado debido a su alto valor nutricional ( $20 \%$ proteína), su rusticidad y su elevada tasa de producción de biomasa y observaciones empíricas hechas por productores en países tropicales reconocen el valor a esta planta como recurso para la producción animal sostenible (Calle y Murgueitio, 2008). También existen reportes sobre su uso como forraje de corte para bovinos de leche y conejos (Ríos, 1999), bovinos en silvopastoreo (Calle y Murgueitio, 2008), en cerdos (Solarte, 1994), en Búfalos (Ríos, 1997) y en ovinos (Vargas, 1992).

En lo que se refiere a los ovinos criollos, se han convertido en un potencial productivo para las familias campesinas debido a su activa participación en el mercado colombiano en el área de carne, cueros y lana de buena calidad. Una de las tantas habilidades de estos animales es su rápida adaptabilidad no solo al clima sino a la topografía de las regiones donde habitan, eficiencia reproductiva, facilidad de crianza, habilidad materna, mansedumbre, además de su alta 
resistencia a enfermedades lo cual hace que sea una especie atractiva para el pequeño productor (García et al., 2008).

De esta manera, según lo establecido por Mahecha y Rosales, (2005) el follaje de Tithonia diversifolia se caracteriza por un buen contenido de nitrógeno total, con alta proporción de nitrógeno de naturaleza aminoacídica, de fósforo, rápida degradabilidad y fermentación a nivel ruminal, baja proporción de nitrógeno ligado a éste y bajo contenido metabolitos secundarios, además se presume la presencia de sustancias pigmentantes. Estos mismos autores realizaron un estudio comparativo con especies forrajeras de amplio uso en la alimentación animal como: Leucaena leucocephala, Gliricidia sepium y Erythrinas, y observaron alta aceptabilidad y respuesta productiva en ovinos, pollos de engorde y gallinas ponedoras, demostrándose la viabilidad de su uso en monogástricos y en rumiantes.

El presente trabajo se realizó con el fin de determinar el efecto de ensilar forraje de botón de oro (Tithonia diversifolia), en diferentes estados de desarrollo (45, 60 y 75 días de corte) y tiempos de maduración del ensilaje (30, 45 y 60 días), sobre la composición bromatológica y digestibilidad in vivo como suplemento en ovinos.

\section{METODOLOGÍA}

El experimento se llevó a cabo, en las instalaciones de la Granja Barcelona en la Unidad de Especies Menores del programa de Medicina Veterinaria y Zootecnia de la Facultad de Ciencias Agropecuarias y Recursos Naturales de la Universidad de los Llanos, la cual se encuentra ubicada en el km 12 vía Puerto López, en el municipio de Villavicencio, Meta. Las características fisiográficas de la región son: altitud de 467 metros sobre el nivel del mar, temperatura promedio de 27 grados centígrados y precipitación promedio anual entre 1830 y $3568 \mathrm{~mm}$ y humedad relativa del $85 \%$ (Roa et al., 2013).

Inicialmente se realizó una poda de nivelación a las plantas, con el fin de dejar un tiempo de recuperación, realizando el primer corte del botón de oro a los 45 días. Posteriormente se procedió a picar el material para la elaboración del ensilaje, al 
cual se le adicionó melaza y agua en proporción 25:75. Se utilizaron canecas con capacidad de almacenar $20 \mathrm{~kg}$ en total del material ensilado (Figura 1), con tiempos de maduración de 30, 45, 60 días; una vez cumplido estos periodos de maduración de los ensilajes, se llevaron al proceso de secado, siendo almacenado cada tratamiento en un frasco rotulado con el respectivo tiempo de corte y maduración del ensilaje, para luego tomar una muestra representativa a la cual se le determinó en el laboratorio de Nutrición Animal de la Universidad, valores de: materia seca (MS), humedad final, extracto etéreo o grasa (EE), proteína (PC), fibra cruda (FC), cenizas, estrato no nitrogenado (ENN) y fibra detergente neutro (FDN). Este mismo procedimiento se realizó en el segundo corte de botón de oro a los 60 días y tercer corte del botón de oro (60 y 75 días) (Tabla 1).

Tabla 1. Tratamientos según días de corte y maduración ensilaje

\begin{tabular}{|c|c|c|c|}
\hline \multirow{2}{*}{ Días de corte } & \multicolumn{3}{|c|}{ Tiempo de maduración en días } \\
\hline & 30 & 45 & 60 \\
\hline 45 & $\begin{array}{c}\mathrm{R}_{1}, \mathrm{R}_{2} \mathrm{y} \mathrm{R}_{3}=5 \mathrm{~kg} \\
\mathrm{~T} 1=45^{\mathrm{C}}: 30^{\mathrm{M}}\end{array}$ & $\begin{array}{c}\mathrm{R}_{1}, \mathrm{R}_{2} \text { y } \mathrm{R}_{3}=5 \mathrm{~kg} \\
\mathrm{~T} 2=45^{\mathrm{C}}: 30^{\mathrm{M}}\end{array}$ & $\begin{array}{c}\mathrm{R}_{1}, \mathrm{R}_{2} \text { y } \mathrm{R}_{3}=5 \mathrm{~kg} \\
\mathrm{~T} 3=45^{\mathrm{C}}: 30^{\mathrm{M}}\end{array}$ \\
\hline 60 & $\begin{array}{l}R_{1}, R_{2} \text { y } R_{3}=5 \mathrm{~kg} \\
T 4=45^{\mathrm{C}}: 30^{\mathrm{M}}\end{array}$ & $\begin{array}{l}R_{1}, R_{2} \text { y } R_{3}=5 \mathrm{~kg} \\
T 5=45^{\mathrm{C}}: 30^{\mathrm{M}}\end{array}$ & $\begin{array}{l}R_{1}, R_{2} \text { y } R_{3}=5 \mathrm{~kg} \\
T 6=45^{\mathrm{C}}: 30^{\mathrm{M}}\end{array}$ \\
\hline 75 & $\begin{array}{c}R_{1}, R_{2} \text { y } R_{3}=5 \mathrm{~kg} ; \\
T 7=45^{\mathrm{C}}: 30^{\mathrm{M}}\end{array}$ & $\begin{array}{c}\mathrm{R}_{1}, \mathrm{R}_{2} \text { y } \mathrm{R}_{3}=5 \mathrm{~kg} ; \\
\mathrm{T} 8=45^{\mathrm{c}}: 30^{\mathrm{M}}\end{array}$ & $\begin{array}{l}R_{1}, R_{2} y R_{3}=5 \mathrm{~kg} \\
T 9=45^{\circ}: 30^{\mathrm{M}}\end{array}$ \\
\hline
\end{tabular}

T: Tratamiento, R: Repetición C: Tiempo de corte planta, M: Tiempo de maduración del ensilaje

Se utilizaron 9 ovinos criollos adultos, de 2 años de edad, con un peso aproximado de $30 \pm 5 \mathrm{~kg}$, fueron 9 tratamientos los cuales se distribuyeron aleatoriamente en jaulas metálicas con comedero, bebedero automático y recolector de excretas y orina, siendo sus dimensiones de $90 \mathrm{~cm}$ de largo, $60 \mathrm{~cm}$ de ancho y $80 \mathrm{~cm}$ de alto (Figura 2).

El trabajo se dividió en una fase de acostumbramiento de 5 días donde a todos los animales diariamente se les suministraba sal mineralizada ad-libitum, $5 \mathrm{~kg}$ forraje fresco picado de kinggrass (Pennisetum purpureun), divido en dos raciones de 2.5 $\mathrm{kg}$ / animal y $400 \mathrm{gr}$ de materia seca (MS) de cada tratamiento. Posteriormente se realizó la fase experimental, que consistió en la de toma de datos (orina excretada cada 12 horas (ml), heces cada 24 horas ( $\mathrm{gr}$ ), consumo por tratamiento ( $\mathrm{gr}$ )) y recolección total de heces (CTH) (Lachmann y Araujo, 1999), medición del peso 
total y recolección de muestras representativas de las mismas (300 g), dichas muestras fueron secadas para su posterior análisis.
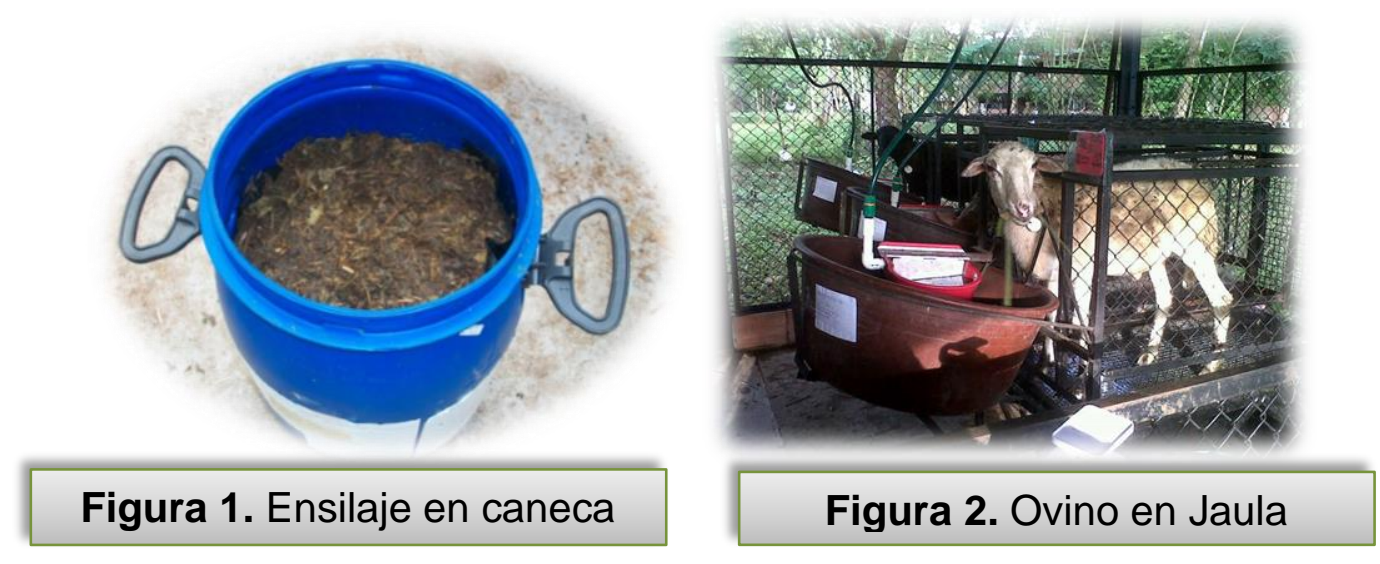

Se realizaron análisis nutricionales en los ensilajes de botón de oro, en el forraje King grass y excretas, donde se determinó el contenido de humedad, cenizas, materia seca, proteína, extracto etéreo, fibra cruda y fibra detergente neutro (AOAC, 2006). Valorando el consumo y la excreción de los nutrientes se determinó el coeficiente de digestibilidad (COD) de todos los nutrientes aplicando las siguientes formulas (Church et al., 2002):

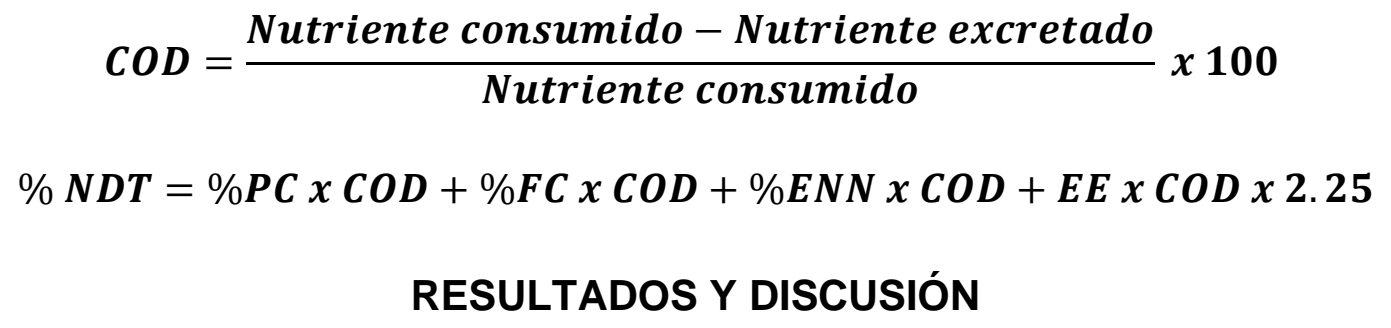

De acuerdo a la información obtenida en este estudio (Tabla 2), correspondiente al ensilaje de botón de oro a los 45 días de corte con 3 tiempos de maduración (30, 45 y 60 días), se observa que los valores más altos en porcentaje de materia seca (MS), humedad $(\mathrm{H})$, grasa (EE) y fibra cruda $(\mathrm{FC})$, corresponde a los 30 días de maduración, aunque las variaciones no son significativas con respecto a los otros tiempo de maduración, por otro lado el rango más alto de proteína se presenta a los 60 días. Al comparar los datos con respecto a los valores de fibra cruda, se observa que la tendencia en el porcentaje de FC es directamente proporcional a 
los días de maduración del ensilaje, es decir se aumenta a medida que avanzan los días de maduración del ensilaje, mientras que la FDN no presenta variaciones por efecto del tiempo.

Los porcentajes de materia seca y ceniza se mantienen en el tiempo, los valores presentan un coeficiente de variación bajo y se comportan de manera uniforme para los distintos periodos de maduración en el estado de floración media (60 días corte). Así mismo, a medida que aumenta la maduración descienden los valores de grasa (EE) en aproximadamente un $0,3 \%$, mientras que en los porcentajes de proteína ( $P C$ ), se obtiene su rango más alto a los 30 días (Tabla 2); estudios realizados por Roa et al., (2010) mostraron valores de proteína de $8 \%$ y $7,2 \%$ a los 30 y 60 días de maduración, comparándolo con los resultados de la Tabla 2 se observa un aumento en promedio de $4 \%$ de la proteína.

Tabla 2. Composición nutricional del ensilaje botón de oro

\begin{tabular}{|c|c|c|c|c|c|c|c|c|c|c|}
\hline \multirow{3}{*}{$\begin{array}{l}\text { Maduración } \\
\text { (días) }\end{array}$} & \multirow{3}{*}{$\begin{array}{l}\text { King } \\
\text { grass }\end{array}$} & \multicolumn{9}{|c|}{ Ensilaje Tithonia diversifolia } \\
\hline & & \multicolumn{3}{|c|}{45} & \multicolumn{3}{|c|}{60} & \multicolumn{3}{|c|}{75} \\
\hline & & T1 & T2 & T3 & T1 & T2 & T3 & T1 & T2 & T3 \\
\hline Nutriente (\%) & & 30 & 45 & 60 & 30 & 45 & 60 & 30 & 45 & 60 \\
\hline Materia Seca & 20,0 & 24,5 & 20,5 & 22,4 & 22,8 & 22,8 & 22,8 & 19,0 & 18,8 & 21,5 \\
\hline Ceniza & 9 & 8,6 & 10,1 & 9,6 & 10,6 & 10,2 & 10,2 & 8,8 & 12,3 & 12,5 \\
\hline Grasa & 1,7 & 2,0 & 1,7 & 1,2 & 0,9 & 0,3 & 0,6 & 1,8 & 1,9 & 1,6 \\
\hline Proteína & 7,6 & 10,1 & 12,8 & 14,1 & 12,3 & 11,9 & 11,5 & 8,4 & 12,1 & 11,0 \\
\hline Fibra Cruda & 39,2 & 16,3 & 2,9 & 3,9 & 3,4 & 5,7 & 9,9 & 5,6 & 1,9 & 1,3 \\
\hline FDN & 57,4 & 24,1 & 25,7 & 25,2 & 23,0 & 27,1 & 33,2 & 23,5 & 29,3 & 26,7 \\
\hline ENN & 36,6 & 49,2 & 58,7 & 59,8 & 59,8 & 57,8 & 49,2 & 64,6 & 67,9 & 68,4 \\
\hline NDT & 53,3 & 58,7 & 66,8 & 68,7 & 66,0 & 63,0 & 55,9 & 70,3 & 76,9 & 76,5 \\
\hline
\end{tabular}

FDN: Fibra detergente neutro, ENN: Extracto no nitrogenado, NDT: nutrientes digestibles totales

Al realizar la comparación de la composición nutricional del ensilaje a los 75 días corte y sus diferentes tiempo de maduración (30, 45 y 60 días) se observa que éste es el que presenta los porcentajes más bajos de MS y $\mathrm{PC}$, con respecto a los periodos anteriores, estos cambios se pueden atribuir a la diferencia entre el tiempo de corte para estos tratamientos siendo mayor para los tres últimos 
tratamientos, donde la planta a los 75 días corte se encuentra en el estado fenológico de floración completa. Para el T7, en donde el valor de FDN se redujo con respecto a los últimos tratamientos su disminución relativa puede suceder cuando algunos forrajes ensilados pueden solubilizar fracciones de hemicelulosas, que hacen parte de la FDN, a lo largo de los procesos de fermentación del ensilado (Cutullic et al., 2011).

La materia seca digestible (DMS) se aumentó en proporción relativa conforme se incrementaban los tiempos de conservación del ensilaje y la edad de corte, pero no se observó efecto de estos dos factores $(P<0,05)$ sobre la DMS, lo que implica que todos los procesos de fermentación fueron totalmente anaeróbicos y no alteraron la disponibilidad de este nutriente, estos resultados resultan superiores a los encontrados por Ribeiro et al., (2007) en el ensilado de girasol (Helianthus annuus L.) con coeficientes de digestibilidad de la materia seca alrededor de $50 \%$. (Tabla. 3). La digestibilidad de la proteína cruda (DPC) fue mayor $(P<0,05)$ a los 75 días de corte en los tres periodos de conservación (T3, T6 y T9) en comparación con los demás tratamientos, presentando los menores valores de DPC $(P<0,05)$ a los 60 días de corte (T7, T8 y T9), sin observar efecto del tiempo de maduración del ensilaje (Tabla 3).

Tabla 3. Parámetros de digestibilidad materia seca, proteína y FDN

\begin{tabular}{cccccccccc}
\hline $\mathbf{C}^{\prime}$ & \multicolumn{3}{c}{ DMS $^{\mathbf{1}}$} & \multicolumn{4}{c}{ DPC $^{\mathbf{3}}$} & \multicolumn{3}{c}{ DFDN $^{\mathbf{3}}$} \\
\cline { 2 - 10 } & $\mathbf{4 5}$ & $\mathbf{6 0}$ & $\mathbf{7 5}$ & $\mathbf{4 5}$ & $\mathbf{6 0}$ & $\mathbf{7 5}$ & $\mathbf{4 5}$ & $\mathbf{6 0}$ & $\mathbf{7 5}$ \\
\hline 30 & $65,6^{\mathrm{aA}}$ & $62,7^{\mathrm{bA}}$ & $70,4^{\mathrm{bA}}$ & $58,5^{\mathrm{aB}}$ & $60,9^{\mathrm{aB}}$ & $73,0^{\mathrm{bB}}$ & $68,4^{\mathrm{aA}}$ & $65,1^{\mathrm{bA}}$ & $75,9^{\mathrm{bA}}$ \\
45 & $64,1^{\mathrm{aB}}$ & $75,1^{\mathrm{bB}}$ & $68,2^{\mathrm{bB}}$ & $55,7^{\mathrm{aB}}$ & $54,5^{\mathrm{aB}}$ & $71,8^{\mathrm{bB}}$ & $61,0^{\mathrm{aB}}$ & $81,4^{\mathrm{bB}}$ & $74,5^{\mathrm{bB}}$ \\
60 & $63,7^{\mathrm{aB}}$ & $73,5^{\mathrm{bB}}$ & $72,9^{\mathrm{bB}}$ & $52,4^{\mathrm{aA}}$ & $67,0^{\mathrm{aA}}$ & $66,4^{\mathrm{bA}}$ & $64,9^{\mathrm{aB}}$ & $79,0^{\mathrm{bB}}$ & $75,1^{\mathrm{bB}}$ \\
\hline
\end{tabular}

Letras superíndices minúsculas diferentes en la misma fila indican significancia estadística en los tiempos de corte $(P<0.05)$. Letras superíndices en mayúsculas diferentes en la misma columna indican diferencias entre niveles de maduración $(P<0.05)$. ${ }^{*} \mathrm{C}=$ Corte desde el rebrote en días. $\mathrm{M}=$ Maduración del ensilaje en días. ${ }^{1}$ Digestibilidad materia seca. ${ }^{2}$ Digestibilidad proteína cruda. ${ }^{3}$ Digestibilidad fibra detergente neutro.

La digestibilidad de la fibra detergente neutro (DFDN), fue menor $(P<0.05)$ a los 45 días de fermentación en los tres tiempos de corte (T1, T4 y T7) exhibiendo efecto de estas dos variables en la degradación de las paredes celulares lo cual implica 
la fermentación de hemicelulosa y celulosa por parte de los microorganismos benéficos como los Lactobacillus, formando ácido láctico y propiónato que son fuentes de glucosa en el metabolismo de rumiantes (Church et al., 2002).

La digestibilidad de los nutrientes digestibles totales (NDT) fue mayor $(\mathrm{P}<0.05)$ para los T7, T8 y T9, observándose un efecto en el tiempo de corte y fermentación, principalmente a los 75 días de corte y 60 de maduración donde T9 presentó el valor más alto $77,7 \%$ con respecto a T8 y T9, lo contario sucedió en los resultados de relación nutritiva (RN), donde el estado de desarrollo de la planta a la cual se le realizó el corte, presentó mejores eficiencias $(P<0.05)$ en el aprovechamiento de la proteína (T1, T2 y T3), es decir a los 30 días de corte en los tres tiempos de maduración, obteniendo valores de 1:6.5, 1:6.8 y 1:5.4, por lo anterior se deduce que por cada kilogramo de proteína digerido por el ensilaje se aprovechó esa cantidad de los demás nutrientes, sumando carbohidratos, grasa y fibra, presentando una relación nutritiva con tendencia a ser media en comparación con los tratamientos en los cuales se realizaron cortes a los 60 días, siendo sus valores de RN bastantes amplios (Tabla 4).

Con relación al pasto King grass (Grafica 1) los parámetros nutricionales en comparación con los de los ensilajes del botón de oro en todas sus épocas de corte y maduración fueron inferiores en cuanto a energía metabolizable, relación nutritiva y nutrientes digestibles totales, presentado un alto contenido de FDN en más de 25 unidades porcentuales con relación a los ensilajes (Tabla 2)

Tabla 4. Parámetros de energía y digestibilidad: relación nutritiva y NDT

\begin{tabular}{cccccccccc}
\hline C / & \multicolumn{3}{c}{$\%$ Digestibilidad } & \multicolumn{4}{c}{$\mathbf{R N}$} & \multicolumn{3}{c}{ NDT } \\
\cline { 2 - 9 } $\mathbf{M}^{\star}$ & $\mathbf{4 5}$ & $\mathbf{6 0}$ & $\mathbf{7 5}$ & $\mathbf{4 5}$ & $\mathbf{6 0}$ & $\mathbf{7 5}$ & $\mathbf{4 5}$ & $\mathbf{6 0}$ & $\mathbf{7 5}$ \\
\hline 30 & $83,7^{\mathrm{aA}}$ & $79,2^{\mathrm{bA}}$ & $86,2^{\mathrm{cA}}$ & $11,1^{\mathrm{aA}}$ & $9,2^{\mathrm{aA}}$ & $9,3^{\mathrm{bA}}$ & $49,9^{\mathrm{aA}}$ & $49,2^{\mathrm{bA}}$ & $61,7^{\mathrm{bA}}$ \\
45 & $82,4^{\mathrm{aB}}$ & $88,7^{\mathrm{bB}}$ & $86,4^{\mathrm{c}}$ & $10,9^{\mathrm{aB}}$ & $18,8^{\mathrm{aB}}$ & $7,5^{\mathrm{bB}}$ & $54,1^{\mathrm{aB}}$ & $64,7^{\mathrm{bB}}$ & $60,6^{\mathrm{bB}}$ \\
60 & $82,2^{\mathrm{aB}}$ & $85,2^{\mathrm{bB}}$ & $72,89^{\mathrm{c}}$ & $11,6^{\mathrm{aC}}$ & $9,7^{\mathrm{aC}}$ & $8,6^{\mathrm{bC}}$ & $48,7^{\mathrm{aB}}$ & $63,8^{\mathrm{bB}}$ & $68,5^{\mathrm{bB}}$ \\
\hline
\end{tabular}

Letras superíndices minúsculas diferentes en la misma fila indican significancia estadística en tiempos de corte $(P<0,05)$. Letras superíndices en mayúsculas diferentes en la misma columna indican diferencias entre niveles de maduración $(P<0,05) .{ }^{*} \mathrm{C}=$ Corte desde el rebrote en días. $\mathrm{M}=$ Maduración del ensilaje en días. ${ }^{1}$ Digestibilidad materia seca. ${ }^{2}$ Digestibilidad proteína cruda. ${ }^{3}$ Digestibilidad fibra detergente neutro 
Por lo anterior, la relación nutritiva de los tratamientos, resulta ser óptima para la alimentación de monogástricos porque se observan valores de RN menores de 6 , como es el caso de T3 $(1: 5,4)$ de lo cual se puede deducir que los cortes deben realizarse cada 30 días, dejando que el ensilaje madure 75 días para que sean apropiados para ser utilizados en cerdos o aves.

Es importante resaltar que a cualquier edad de corte y tiempo de maduración del ensilaje con el que se suplementó la dieta base de King grass, es favorable para la alimentación rumiantes como ovinos y bovinos. La digestibilidad de MS representa una buena estimación del grado en que un ingrediente es digerido y absorbido por el tracto digestivo, para este estudio, los coeficientes estuvieron por encima del $50 \%$. La DFDN aumenta en relación a la edad de la planta. En cuanto a la DPC, esta se ve afectada conforme incrementa el tiempo de corte de la planta del ensilaje, entre mayor es su estado de desarrollo mayor será la cantidad de proteína aprovechada por el animal pero un mayor tiempo de maduración del ensilaje mantiene o aumenta su digestibilidad.

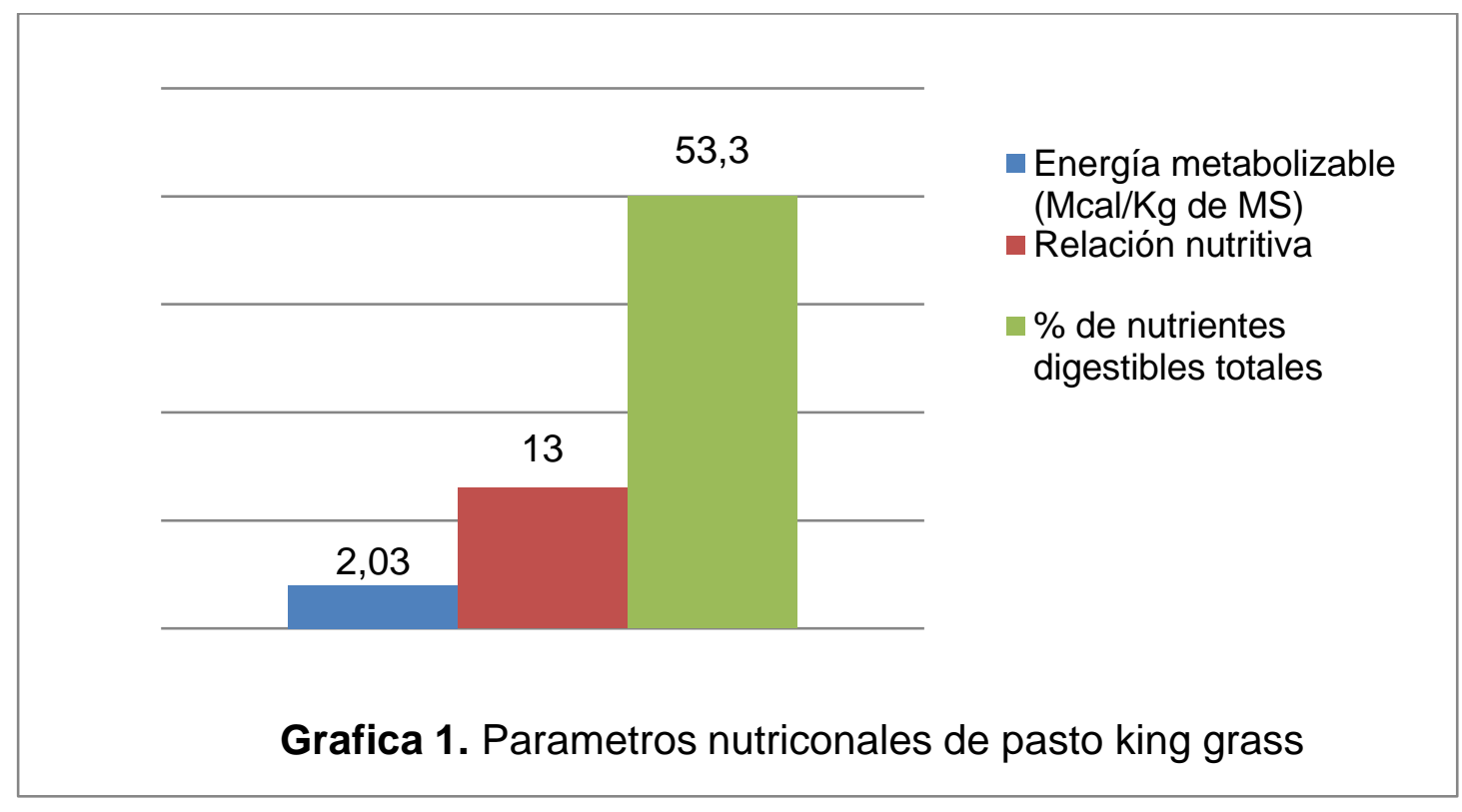

Se establece que la calidad nutricional del ensilaje, arroja valores favorables de materia seca (20 a 25\%), proteína (12\%), y contenidos de FDN por debajo de $40 \%$, se sugiriere la posibilidad de ser incluidos en planes de alimentación y suplementación de vacas lecheras favoreciendo el consumo de materia seca, ya 
que el contenido de FDN se relaciona positivamente con el llenado del rumen y con la densidad del forraje, y la velocidad de paso de las fracciones no digeribles en rumen.

\section{CONCLUSIONES}

De acuerdo con los resultados investigativos y requerimientos nutricionales de la especie, se desprende que el ensilaje de botón de oro asociado a king grass, se presenta como una alternativa de alimentación para ovinos siempre que se disponga del forraje, sugiriendo que el estado de desarrollo óptimo para el corte del forraje y su posterior ensilado resulta ser a los 60 días, ya que presenta los rangos más altos de disponibilidad y aprovechamiento de nutrientes, por otro lado los días de maduración del ensilaje en sus tres etapas resultan ser favorables sin generar pérdidas nutricionales en la calidad inicial del forraje conservado.

Los resultados obtenidos en este trabajo generan línea base como punto de partida para tomar decisiones o adelantar estudios de evaluaciones posteriores en otras especies, en corroborar la calidad nutricional favorable que tiene este tipo de recurso.

\section{BIBLIOGRAFÍA}

1. Association Official Methods of Analysis (AOAC). (18 $\left.8^{\text {th }}\right)$ Association of Official Analytical Chemists, Arligton, VA. Washington, D.C. 2006.

2. Barahona R. y Sánchez S. Limitaciones físicas y químicas de la digestibilidad de pastos tropicales y estrategias para aumentarla. Revista CORPOICA, 6 (1): 69-82. 2005.

3. Chacón P., Vargas C. Consumo de Pennisetum purpureum cv. King Grass a tres edades de cosecha en caprinos. Revista Agronomía Mesoamericana, 21 (2): 267-274. 2010.

4. Church D., Pond W., Pond K. Fundamentos de nutrición y alimentación de animales, segunda edición, UTHEA WILEY, Ed. Limusa, México D.F, México, 635 p. 2002.

5. Calle D., Murgueitio E. El botón de oro: arbusto de gran utilidad para sistemas ganaderos de tierra caliente y de montaña. Federación Colombiana de Ganaderos. Carta Fedegan, N. 108, p 54-62. 2008.

6. Cuadrado C., Mejía S. H., Contreras A. Manejo Agronómico de Algunos Cultivos de Forrajeros y Técnicas para su Conservación en la Región Caribe Colombiana. Manual Técnico. Cerete, Córdoba: CORPOICA, Agosto de 2003. 
7. Cutullic E., Delaby L., Gallard Y. Disenhaus C. Dairy cows' reproductive response to feeding level differs according to the reproductive stage and the breed. Animal, 5 (5): 731-740. 2011.

8. Escobar J., Suárez J., Restrepo L., Mahecha L. Uso de Tithonia diversifolia (Hemsl.) Gray (Botón de oro) como reemplazo parcial del alimento. Revista Colombina de Ciencias Pecuarias, 16 (3 Suplemento): 28. 2003.

9. Espinoza F., Argenti P., Gil J. L., León L., Perdomo E. Evaluación del pasto king grass (Pennisetum purpureun Cv. King Grass) en asociación con leguminosas forrajeras. Revista Zootecnia Tropical, 19 (1): 59-71. 2001.

10. Figueredo B. L., del Toro M. I. Los ovinos. Una producción de bajos insumos. Revista Electrónica de Veterinaria - REDVET, 6 (9): Art. 10. 2005.

11. Flores G., Gonzales A., Castro J., Castro P., Cardelle M., Fernández B., Valladares J. Evaluación de métodos de laboratorio para la predicción de digestibilidad in vivo de la materia orgánica de ensilajes de hierba y planta entera de maíz. Sociedad Española para el Estudio de los Pastos, 33 (1): 199. 2003.

12. Fonseca F. N. Contribución al estudio de la alimentación del ovino pelibuey cubano. Tesis de grado Doctor en Ciencias Veterinarias, Universidad de Granma, Instituto de Ciencia Animal. Cuba. 161 p. 2003.

13. Garcés M., Adelaida L. Ensilaje como fuente de alimentación para el ganado. Revista Lasallista de Investigación, 1 (1): 66-71. 2004.

14. García D. E., Medina M. G., Cova L. J., Soca M., Pizzani P., Baldizán A., Domínguez C. E. Aceptabilidad de follajes arbóreos tropicales por vacunos, ovinos y caprinos en el estado Trujillo, Venezuela. Revista Zootecnia Tropical, 26 (3): 191-196. 2008.

15. Hernández C. A. Utilización de la morera (Morus alba) y Botón de oro. Tesis Pregrado, Universidad Michoacana de San Nicolás de Hidalgo, Morelia Michoacán, 2011.

16. Lachmann M., Araujo F. La estimación de la digestibilidad en ensayos con rumiantes. Universidad del Zulia, Facultad de Ciencias Veterinarias. Maracaibo-Venezuela, $1999 . \quad$ Disponible http://www.secalc.ula.ve/AUPA/docuPDFS/digestibilidadderumiante.pdf

17. Mahecha L., Rosales M. Valor nutricional del follaje de botón de oro (Tithonia diversifolia (Helmsl.) Gray, en la producción animal en el trópico. Livestock Research for Rural Development. 17 (9): Art. 100. 2005.

18. Maza A. L., Vergara G. O., Paternina E. Evaluación química y organoléptica del ensilaje de maralfalfa (Pennisetum sp.) más yuca fresca (Manihot esculenta). Revista MVZ Córdoba, 16 (2): 2528- 537. 2011.

19. Medina M. G, García D. E., Gonzales M. E., Cova L. J. Moratinos P. Variables morfoestructurales y de calidad de la biomasa de Tithonia diversifolia en la etapa inicial de crecimiento. Revista Zootecnia Tropical, 27 (2): 121-134. 2009.

20. Mrad de Osorio, A. Ética en la investigación con modelos animales experimentales. Alternativas y las $3 \mathrm{RS}$ de Russel. Una responsabilidad y un compromiso ético. Revista Colombiana de Bioética, 1 (1): 163-183. 2006.

21. Naranjo J. F., Cuartas C. A. Caracterización nutricional y de la cinética de degradación ruminal de algunos de los recursos forrajeros con potencial para 
la suplementación de rumiantes en el trópico alto de Colombia. Revista CES Medicina Veterinaria y Zootecnia. 6 (1): 9-19. 2011.

22. Nieves D., Terán O., Cruz L., Mena M., Gutiérrez F., Julio L. Digestibilidad de nutrientes en follaje de árnica (Tithonia diversifolia) en conejos de engorde. Tropical and Subtropical Agroecosystems, 14 (1): 309-314. 2010.

23. Pérez A., Montejo I., Iglesias J. M., López O., Martín G. J., García D. E., Idolkis M. Hernández A. Tithonia diversifolia (Hemsl.) A. Gray. Revista Pastos y Forrajes, 32 (1): 1-15. 2009.

24. Ríos C. I. Botón de oro Tithonia diversifolia (Hemls.) Gray en Árboles y arbustos forrajeros utilizados en alimentación animal como fuente proteica. 2da edición. Colciencias - CIPAV. Cali, Colombia, p 115-126. 1997.

25. Ríos C. I. Tithonia diversifolia (Hemsl.) Gray, una planta con potencial para la producción sostenible en el trópico. En: Agroforestería para la producción animal en América Latina. Estudio FAO Producción y Sanidad Animal N. 143. FAO, Roma. Eds. Sánchez, M. D. y Rosales, M. p 217-230. 1999.

26. Roa M. L., Castillo C. A., Téllez E. Influencia del tiempo de maduración en la calidad nutricional, de ensilajes con forrajes arbóreos. Revista Electrónica Sistemas de Producción Agroecológicos, 1 (1): 63-73. 2010.

27. Ribeiro P. L., Goncalves L., Rodríguez N., Tomich T. Ensilaje de Girasol como Opción Forrajera. Brasil: Jornada sobre Producción y Utilización de Ensilajes, p 31-50. 2007. Disponible En: http://www.cpatsa.embrapa.br/public eletronica/downloads/OPB1719.pdf

28. Segura F. A., Patiño A. C. Delignificación selectiva del pasto king grass usando basidiomicetos ligninolíticos. Revista Salud Pública y Nutrición, Ed. Especial N. 11: 1-5. 2006.

29. Solarte A. Experiencias de investigación participativa en sistemas de Producción Animal en dos zonas del Valle del Cauca. Memorias III Seminario Internacional Desarrollo Sostenible de Sistemas Agrarios. Cali, Colombia. p 49-72. 1994

30. Verdecia D., Ramírez J., Loenard I., Álvarez Y., Bazán Y., Bodas R., Andrés S., Álvarez J., Giráldez F. y López S. Calidad de la Tithonia diversifolia en una zona del Valle del Cauto. Revista Electrónica de Veterinaria REDVET, 12 (5): Art. 3. 2011.

31. Valencia T. L., Restrepo P. J., Ceron H. D. Determinación de la digestibilidad in vivo en ovinos utilizando dietas a base de forrajes tropicales. Revista de Investigación Agraria y Ambiental, 1 (1): 25-29. 2010.

32. Vargas J. E. Evaluación de la aceptación del botón de oro en la dieta de las ovejas de pelo. III Seminario Internacional. Desarrollo sostenible de sistemas agrarios. Colombia. p 135. 1992.

33. Wanjau S., Mukalama J., Thijssen R. Transferencia de biomasa: Cosecha gratis de fertilizante. LEISA, Revista de Agroecología. 13 (3): 25. 1998. 